\title{
Article \\ Study on Two-Phase Mixing Inside Flow Focusing/Blurring Nozzle by Gray Distribution Analysis Method
}

\author{
Jin Zhao ${ }^{1,2, *}$, Zhi Ning ${ }^{1,2, *}$, Ming L $\ddot{u ̈}^{1,2}$ and Chunhua Sun ${ }^{1,2}$ \\ 1 School of Mechanical, Electronic and Control Engineering, Beijing Jiaotong University, Beijing 100044, China; \\ lvming@bjtu.edu.cn (M.L.); $11116347 @ b j t u . e d u . c n$ (C.S.) \\ 2 Beijing Key Lab Powertrain New Energy Vehicle, Beijing Jiaotong University, Beijing 100044, China \\ * Correspondence: 15121365@bjtu.edu.cn (J.Z.); zhining@bjtu.edu.cn (Z.N.)
}

Citation: Zhao, J.; Ning, Z.; Lü, M.; Sun, C. Study on Two-Phase Mixing Inside Flow Focusing/Blurring Nozzle by Gray Distribution Analysis Method. Appl. Sci. 2021, 11, 6260. https://doi.org/10.3390/app11146260

Academic Editor: N.C. Markatos

Received: 11 May 2021

Accepted: 2 July 2021

Published: 6 July 2021

Publisher's Note: MDPI stays neutral with regard to jurisdictional claims in published maps and institutional affiliations.

Copyright: (c) 2021 by the authors. Licensee MDPI, Basel, Switzerland. This article is an open access article distributed under the terms and conditions of the Creative Commons Attribution (CC BY) license (https:// creativecommons.org/licenses/by/ $4.0 /)$.

\begin{abstract}
The application prospect of a flow focusing/blurring nozzle is broad but research on gas-liquid flow inside the nozzle is not comprehensive. The gas-liquid mixing inside the nozzle is difficult to study by visualization experiment, so this paper proposes to study the gas-liquid flow or mixing inside the nozzle by the gray scale level distribution of the experimental images. The results show that the increase of air flow rate is beneficial to two-phase mixing inside the nozzle, while the influence of water flow rate, tube hole distance (the distance between inner tube and nozzle outlet) and orifice diameter increase is opposite. The influence of air inertia force on two-phase mixing is weaker than the water inertia force under different parameters, the effect of the air inertia force on two-phase mixing is similar to tube hole distance under a small flow rate, the effect of the orifice diameter on two-phase mixing is relatively weak. In addition, the analysis of the gas-liquid flow field in the mixing zone shows that the gas-liquid flow in the nozzle is stable in the flow focusing mode. In the flow blurring mode, the gas-liquid flow inside the nozzle has radial stability but axial pulsation. In the transition mode, the gas-liquid flow inside the nozzle is unstable, but the gas-liquid flow is close to the flow blurring mode.
\end{abstract}

Keywords: flow focusing/blurring nozzle; two-phase flow and mixing; experimental study; gray scale level distribution

\section{Introduction}

The flow focusing/blurring nozzle is a two-phase nozzle developed by GañánCalvo [1,2]. Although this nozzle is simple in structure, it is effective in producing droplets and atomizing. In recent years, this nozzle has been widely used in various industrial applications, including biological medicine, high viscosity liquid atomization, multiphase combustion and industrial painting [3].

When the flow parameters or nozzle structure change, the nozzle will show two different modes: flow focusing and flow blurring. In the flow focusing mode, the nozzle is more likely to produce micro-nano droplets. Gañán-Calvo conducted experimental and theoretical research on the flow focusing nozzle [4-6] and presented the relationship between nozzle inlet pressure, flow rate, jet diameter and droplet diameter. Herrada gave the influence relationship between the recirculation cell and the dripping regime by numerical simulation [7]. Si Ting further divided the flow focusing into six modes and studied the characteristics and causes of each mode [8]. A large number of research results enable people to have a deep understanding of flow focusing so that it can be widely used in the biological medicine field.

In the flow blurring mode, the nozzle is more widely used in the liquid atomization and combustion. Many researchers used experimental methods to study the atomization effect of the nozzle. The results show that the nozzle has a good atomization effect on the liquid, even if the viscosity of the liquid is high [9-12]. However, due to the small size of the nozzle, it is difficult for experimental equipment to observe the two-phase flow inside 
the flow blurring nozzle, so the research on the working principle of the flow blurring nozzle is not comprehensive, which limits the further application of this nozzle in the atomization and combustion field.

In recent years, image processing and analysis technologies have developed rapidly, and they have been well applied in multiphase flow and mixing fields. Qingtai Xiao studied two-phase mixing in a direct contact boiling heat transfer process, by using the gray distribution of the images [13-15]. The results show that the gray distribution of the experimental images are related to the degree of two-phase mixing. Wang chong made a comparative study on the gray distribution of different cross sections of the two-phase mixing images in the mixer and believed that the gray distribution of experimental images could describe the two-phase mixing [16]. Yang Lin's research results showed that the gas flow rate at the mixer inlet would change the bubble distribution and thus affect the gray distribution of the experimental images [17]. Xiangru Li [18,19] and Semlitsch [20] also studied the unstable mode of underexpanded jets and the generation mechanism of screech harmonics in supersonic jets by using shadowgraph imaging methods similar to the gray distribution analysis method. These research objects are similar to the gas-liquid flow in the mixing zone of the flow blurring nozzle. Therefore, it is feasible to use the gray distribution of the experimental images to study the gas-liquid flow in the mixing zone of the flow focusing/blurring nozzle.

In this paper, the gas-liquid interaction in the mixing zone of the flow focusing/blurring nozzle was analyzed and studied by using the gray distribution of the experimental images. We compared the gray distribution of two-phase flow images in the mixing zone under different conditions and analyzed the feasibility of using this method to study the two-phase flow in the mixing zone of the flow focusing/blurring nozzle. Using the gray distribution of the experimental images, we analyzed the gas-liquid's mixing intensity and uniformity in the mixing zone and studied the flow characteristics of the local flow field.

\section{Materials and Methods}

\subsection{Atomizer}

A simplified modular flow focusing/blurring nozzle was developed for the experiment. The nozzle is composed of three modules: liquid-phase delivery inner tube (A), nozzle support body (B) and two-phase mixing part (C), and its structure section view and real object are shown in Figure 1.

(D)

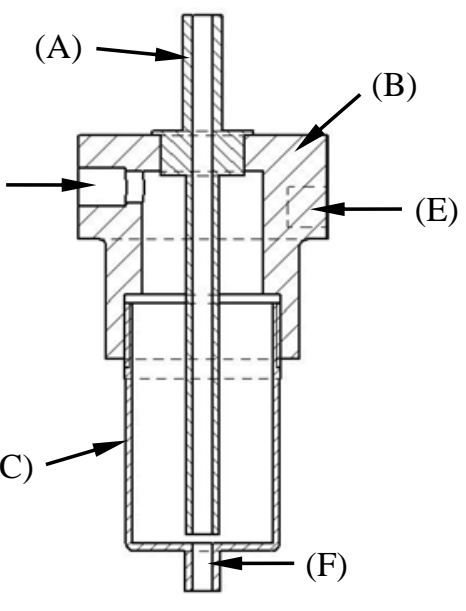

(a) Section view of the nozzle

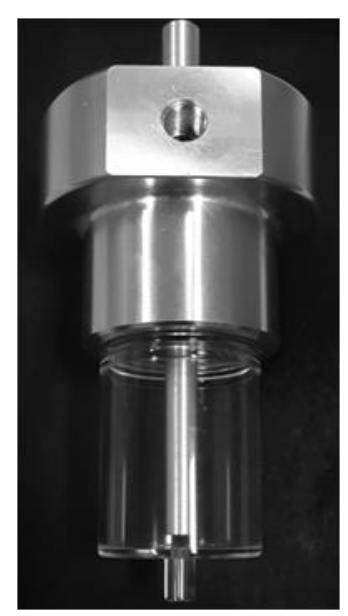

(b) Real object

Figure 1. Structure and composition of the flow focusing/blurring nozzle.

The liquid-phase delivery inner tube and nozzle support body were made of aluminum alloy, and gas-phase inlet (D) and fixing bolts (E) were placed at the nozzle support 
body. The two-phase mixing part was made of plexiglass to allow the visualization of the flow in the mixing zone and has a spray hole (F) at the bottom.

The three modules of the flow focusing/blurring nozzle are connected by pipe threads. The sizes of key components are determined by referring to the relevant literatures and preliminary experiments, as shown in Table 1 . The selection of nozzle structure parameters in Table 1 can not only ensure the clarity of the gas-liquid flow images inside the nozzle but also meet the needs of research on the influence of nozzle structure parameters.

Table 1. Structural parameters of the experimental flow focusing/blurring nozzle.

\begin{tabular}{ccc}
\hline Parameter & Symbol & Value/mm \\
\hline Nozzle inner tube diameter & $D$ & 5 \\
Tube hole distance & $H$ & $1 / 2 / 3 / 4 / 5$ \\
Orifice diameter & $D_{h}$ & $3 / 5 / 7$ \\
Hole length & $l_{h}$ & 10 \\
\hline
\end{tabular}

\subsection{Experimental Platform}

A schematic diagram of the experimental platform is shown in Figure 2. When the gas-liquid mixing inside the nozzle is studied, the properties of water and common liquid working fluids (such as gasoline) are similar. Therefore, on the basis of experimental safety and environmental protection, water and air were selected as the working fluid and atomizing gas, respectively. The liquid was injected into the nozzle from a water tank through a rotameter and check valve. The gas path is similar to the liquid path but the power source is an air compressor. The water and air flow rates were measured by rotameters and the parameters and adjustment range of the experimental equipments are labeled in Figure 2. The adjustment range of gas and liquid flow rates is determined by pre experiment. In the flow rate range selected in the Figure 2, the flow focusing/blurring experimental nozzle designed by us can work in different modes according to the needs.

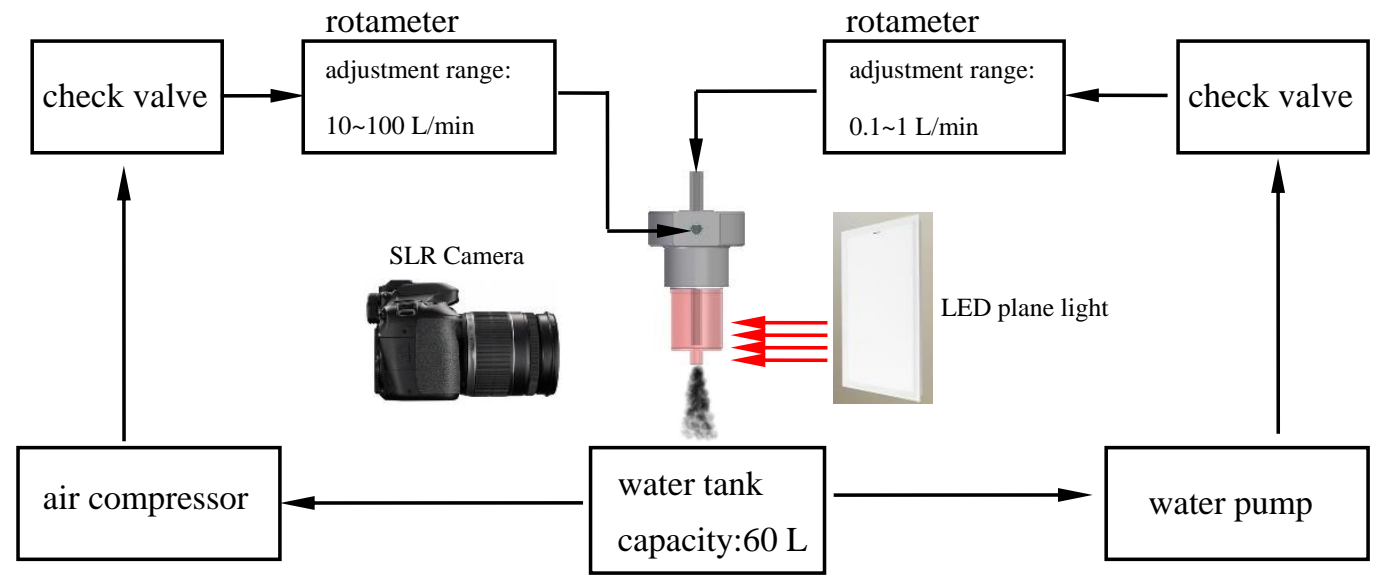

Figure 2. Schematic diagram of experimental platform.

A white LED plane light was used as the experimental light source to obtain two-phase flow images. The plane light is a surface light source, this kind of light source has soft light, uniform illumination, small contrast between light and dark, and shadows do not easily appear in the image. The use of a plane light can effectively reduce the impact of plexiglass and fluid refraction on visualization. The gray distribution analysis method requires high definition of the experimental images. In the flow blurring mode, the gas-liquid flow in the nozzle changes rapidly with time and a relatively clear image can be obtained by choosing a smaller shutter speed. Therefore, the images of the two-phase flow were captured using a SLR camera (Canon 80D), which supported images with 24 million pixels and had a shutter speed of $1 / 8000$ s. 
When we do each set of experiments, the air injection and liquid supply flow rates were adjusted to form different two-phase flow conditions. The water pump and air compressor were turned on such that the two fluids were mixed. The pipelines were checked to ensure that no leakage occurred and then the gas and liquid flow rates were adjusted slowly and smoothly to the experimentally specified value. When the experimental conditions reached a steady state for about $1 \mathrm{~min}$, the experimental data, including the air and liquid flow rates, and two-phase flow images, were recorded using the facilities and instruments described previously.

\section{Gray Distribution Analysis of Experimental Images}

\subsection{Gray Distribution of Image}

The experimental images of the gas-liquid flow in the mixing zone are composed of a large number of pixels. The gray distribution of the experimental images can be obtained by counting the light and shade degree of each pixel. Therefore, the gray distribution is actually the statistical result of the brightness and darkness of the experimental image.

When the working conditions of the nozzle change, as the gas-liquid flow pattern in the nozzle gradually changes from flow focusing to flow blurring, the liquid cone in the mixing zone gradually distorts and finally breaks, and the mixing degree of gas-liquid two-phase fluid increases. Under different conditions, the distribution of the gas and liquid fluid in the mixing zone is different, and the density and other physical parameters of the fluid in different positions of the mixing zone are different. The most important thing is that the size, quantity and distortion degree of the gas-liquid interface in different positions of the mixing zone are different. The difference of these parameters makes the transmission, refraction and reflection ability of the gas-liquid flow to the light at different positions in the mixing zone different, resulting in different gray distribution of the experimental images. This makes it possible to use the gray distribution of the experimental images to study the two-phase flow in the mixing zone under different conditions.

In this section, the basic concept of the gray distribution of the experimental images is described. And in Section 3.2, the relationship between the gray distribution of the experimental images and the flow patterns (flow characteristics) inside the nozzle is qualitatively analyzed through several cases. Through the qualitative relationship between the flow patterns (flow characteristics) and the gray distribution curves, we can study the gas-liquid flow and mixing inside the flow focusing/blurring nozzle.

For each pixel, the gray scale is divided into 0 to 255 , total 256, gray levels. Close to 0 level gray, indicating that the image is dark; close to 255 level gray, indicating that the image is bright. Therefore, for an experimental image $I(M, N)$, its gray histogram can be expressed as [21]:

$$
h\left(r_{k}\right)=n_{k} \text { for } k=0,1,2, \ldots, L
$$

where $r_{k}$ is the $k$ level gray of the image $I(M, N), n_{k}$ is the number of pixels in image $I(M, N)$ with grayscale value $r_{k}$, and $L$ is the largest gray level of all images.

When comparing the gray distribution of different images, the image size will have a significant impact on the number of pixels which will reduce the accuracy of the gray histogram. In order to avoid the influence of the image size, we defined the normalized gray histogram as [21]:

$$
\mathrm{P}\left(r_{k}\right)=\frac{h\left(r_{k}\right)}{M N}=\frac{n_{k}}{M N} \text { for } k=0,1,2, \ldots, L
$$

where $M$ and $N$ are the number of pixels in the row and column of the image, respectively. The components of $\mathrm{P}\left(r_{k}\right)$ are estimates of the frequency of gray levels occurring in an image.

In this paper, the gray histogram and normalized gray histogram of experimental images are obtained by Matlab software. At present, the research results on the gas-liquid flow in the mixing zone of the flow focusing/blurring nozzle using the gray distribution have not been reported. Therefore, the feasibility of using the gray distribution to study the gas-liquid flow in the mixing zone will be analyzed below. 


\subsection{Feasibility Analysis}

For the flow focusing/blurring nozzle, when the flow parameters and the nozzle structure are different, the two-phase interaction in the mixing zone is different, so the flow pattern will be different. Different flow patterns will lead to different gray distribution of the experimental image. In this part, we will analyze the change of the gray distribution of the experimental image with time and study whether the gray distribution of one experimental image can represent the gray distribution of the experimental images under a certain working condition. Then, we will choose several cases. By comparing the difference of the gas-liquid flow images in mixing zone and the difference of the gray distribution of the experimental images in these cases, we can give the relationship between the gray distribution of the experimental images and the flow patterns (flow characteristics), and verify the feasibility of gray distribution analysis methods to study gas-liquid flow inside the nozzle, to a certain extent.

Figure 3 shows the gray distribution curves of the experimental images in a mixing zone at different times when the gas flow rate is $40 \mathrm{~L} / \mathrm{min}$, the liquid flow rate is $600 \mathrm{~mL} / \mathrm{min}$, the orifice diameter is $5 \mathrm{~mm}$, and the tube hole distance is $1 \mathrm{~mm}$.

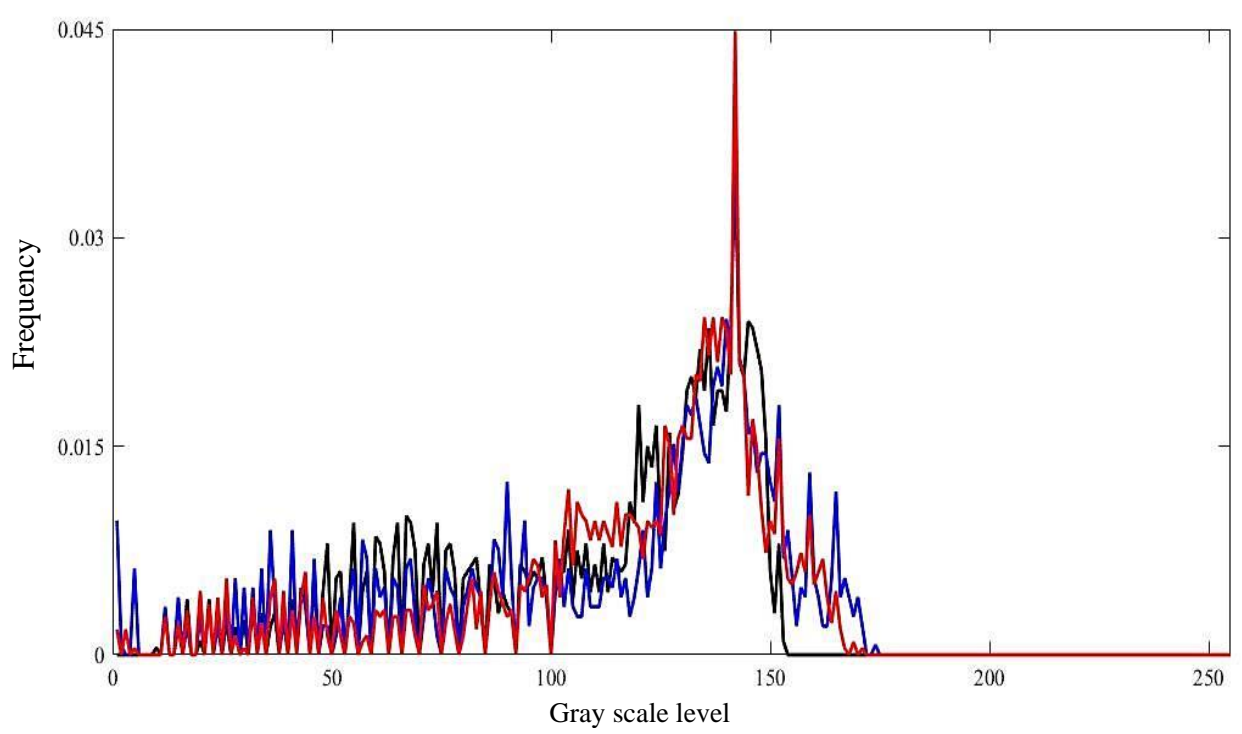

Figure 3. The gray distribution curves of two-phase flow images under different times.

It can be seen from Figure 3 that the gray distribution of the experimental images of gas-liquid flow inside the nozzle has certain differences at different times. This difference may be caused by the high frequency instability of the turbulent flow. The effect of high frequency turbulence instability can be reduced to a certain extent by averaging the gray distribution of the experimental images at different times under the same condition, but it cannot be completely eliminated. The average value of the experimental image's gray distribution curve is also affected by many parameters (such as the number of the experimental images). When we study the gas-liquid mixing in the nozzle through the gray distribution of the experimental images, we pay more attention to the overall trend of the gray distribution curve and we can see from Figure 3 that the difference of the gray distribution curve caused by high-frequency turbulence instability has little effect on the overall trend of the gray distribution curve. Therefore, for one working condition, it is feasible to use the gray distribution of one experimental image to study the gas-liquid mixing in the nozzle. Figure 3 also shows that the analysis results of the gray distribution analysis method for gas-liquid flow in the nozzle are statistically convergent.

Figure 4 shows the images and gray distribution of different flow patterns in the mixing zone obtained by the experiment. The gas-liquid flow images in the mixing zone of the nozzle are marked with a red dotted rectangular box, which is also the area to extract the gray 
distribution of the experimental image. In the experimental images of Figures 5 and 6, the extracted area of the gray distribution curve is also labeled.

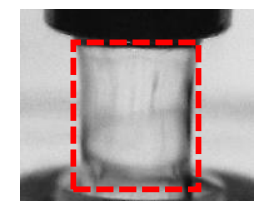

(a) Case 1 (flow focusing)

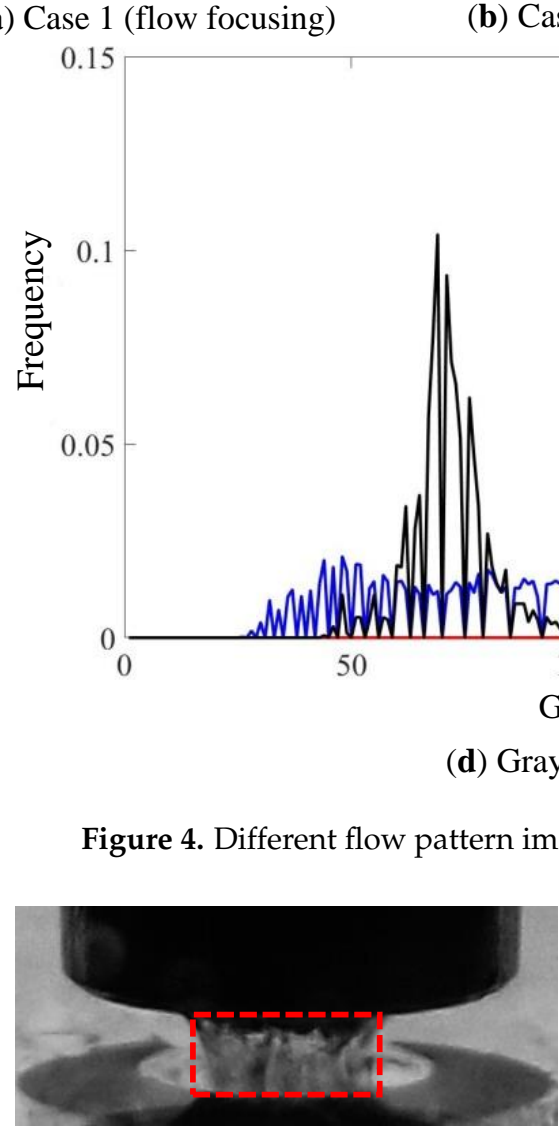

(a) Case 1 (flow blurring)

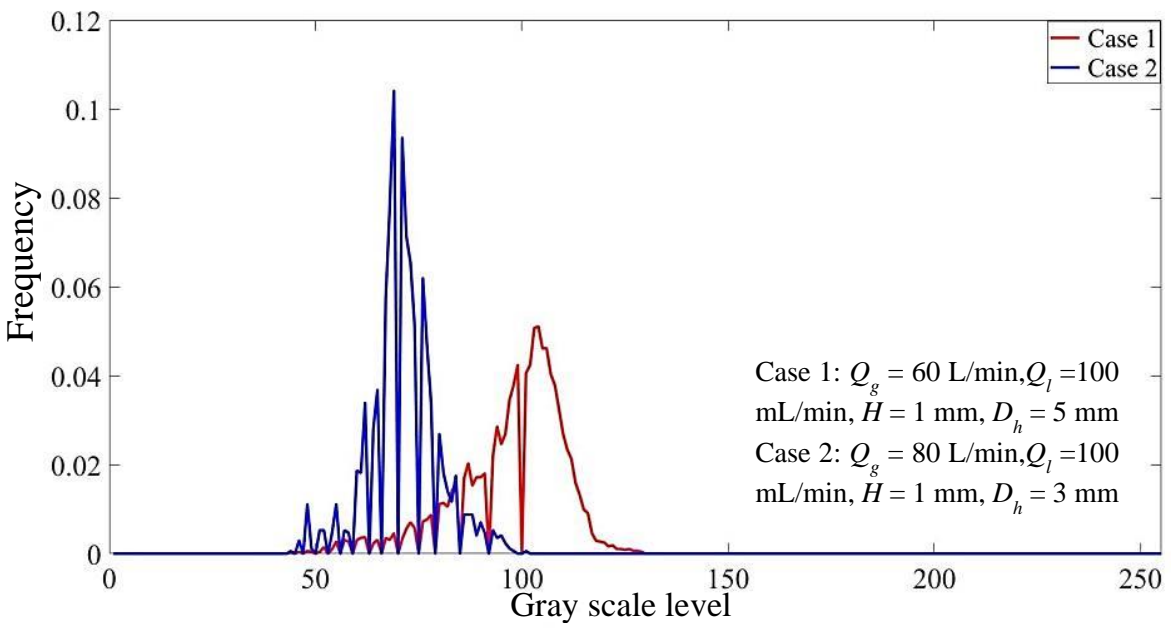

Case 1: $Q_{g}=5 \mathrm{~L} / \mathrm{min}, Q_{l}=800$ $\mathrm{mL} / \mathrm{min}, H=5 \mathrm{~mm}, D_{h}=7 \mathrm{~mm}$ Case 2: $Q_{g}=80 \mathrm{~L} / \mathrm{min}, Q_{l}=100$ $\mathrm{mL} / \mathrm{min}, H=1 \mathrm{~mm}, D_{h}=7 \mathrm{~mm}$ Case 3: $Q_{g}=80 \mathrm{~L} / \mathrm{min}, Q_{l}=100$ $\mathrm{mL} / \mathrm{min}, H=1 \mathrm{~mm}, D_{h}=3 \mathrm{~mm}$

Gray scale level d) Gray scale level distribution

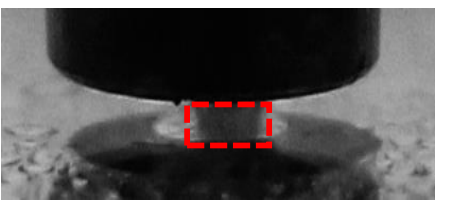

(c) Case 3 (flow blurring) 


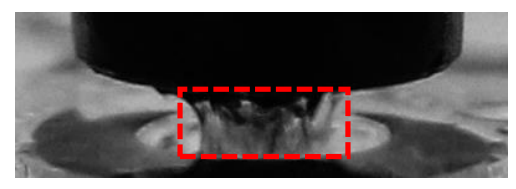

(a) Case 1 (flow blurring)

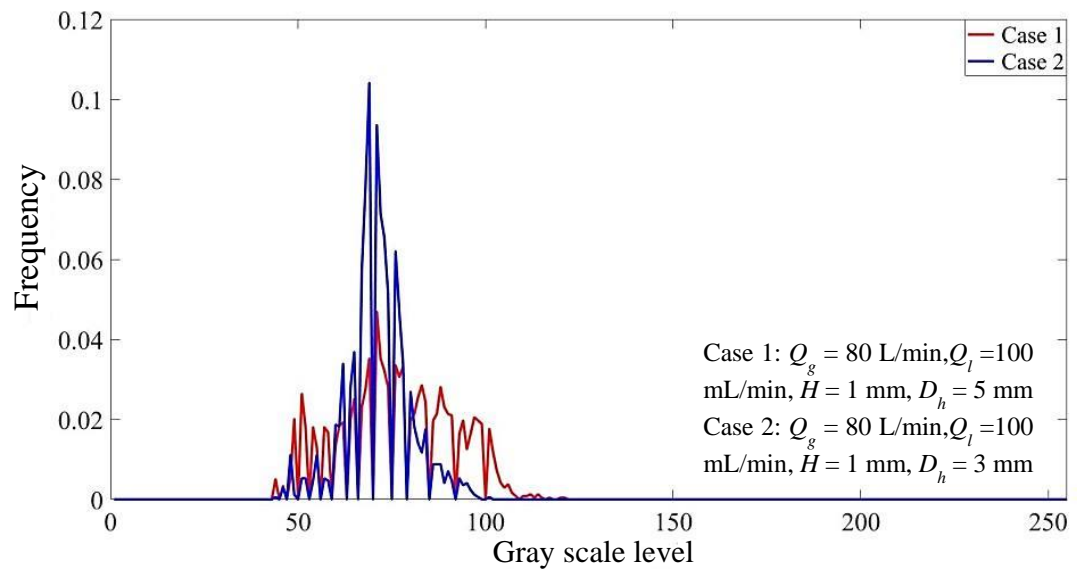

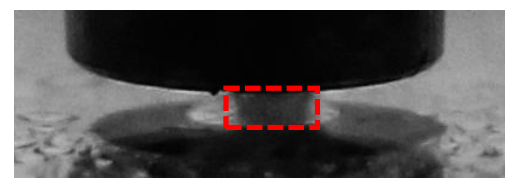

(b) Case 2 (flow blurring)

(c) Gray scale level distribution

Figure 6. The experimental image with different mixing uniformity and its gray distribution.

As can be seen from Figure 4, for case 1, the gas-liquid two-phase fluids in the mixing zone do not mix; we can observe the liquid column in the mixing zone, and the boundary of the gas-liquid two-phase fluids is relatively clear, so the flow pattern in the mixing zone is flow focusing. At this time, the gas-liquid interaction in the mixing zone is weak, the light enters the SLR camera through transmission and the brightness of the pixel points is high and uniform, so the image has a high gray level and a good concentration. For case 3, the boundary of the gas-liquid two-phase fluids in the mixing zone is fuzzy, the gas-liquid flow in the mixing zone is close to fog and the gas-liquid mixing is good, so the flow pattern in the mixing zone is flow blurring. At this time, the liquid in the mixing zone is broken into tiny droplets by gas, and the light enters the SLR camera by scattering. The brightness of the pixels is low but still relatively uniform, which reduces the gray level of the image, but the concentration of gray distribution is still very good.

In case 2, the boundary of the gas-liquid two-phase fluids in the mixing zone is also fuzzy. The gas-liquid fluids mixed, but it is obvious that the gas-liquid flow in the mixing zone is not uniform. The flow pattern in the mixing zone is in a transition state between flow focusing and flow blurring. At this time, there are both liquid drops and distorted twophase interface in the mixing zone, which makes the light enter the SLR camera through transmission or scattering. Therefore, the pixel points of the image are either bright or dark, which is very nonuniform, resulting in a large range of gray distribution of the image and a smooth gray distribution curve.

Figure 4 and its analysis show that the gray distribution of the experimental image is different when the flow pattern of the mixing zone of the flow focusing/blurring nozzle is different, so it is completely feasible to use the gray distribution of the two-phase flow images to identify the flow pattern. In fact, there are many methods to distinguish the flow pattern in the mixing zone, and the two-phase flow in the mixing zone under the flow blurring is the real difficulty in the study of this nozzle. Generally, the mixing intensity and uniformity can be used to characterize the two-phase mixing in the mixing zone under the flow blurring, but there is no suitable instrument to measure these two parameters. The gray distribution of the experimental images can be used to analyze the gas-liquid mixing intensity and uniformity. Figures 5 and 6 show the experimental images with different mixing intensity or uniformity and their gray distribution curves, respectively. 
Figure $5 \mathrm{a}, \mathrm{b}$ show the two-phase flow images in the mixing zone with different mixing intensity. Compared with case 1, the gas-liquid flow in the mixing zone is closer to fog in case 2, and the boundary of the gas-liquid fluid is more fuzzy, which indicates that the gas-liquid mixing intensity in case 2 is stronger. By comparing the gray distribution of case 1 and case 2, it can be found that the average gray scale level of the images is lower when the mixing intensity of two-phase is larger. This is because, when the gas-liquid mixing intensity is relatively high the number of broken droplets is large and the size of the droplets is small, which makes the scattering effect stronger. Therefore, the light entering the image acquisition equipment is weakened and the average gray level of the image is relatively low.

Figure $6 \mathrm{a}, \mathrm{b}$ show the two-phase flow images in the mixing zone with different mixing uniformity. Compared with case 2, the gray scale level of the gas-liquid flow images at different positions in the mixing zone in case 1 is significantly different, which indicates that the uniformity of the gas-liquid fluid mixing in the mixing zone in case 1 is low. The gray distribution of the images indicates that the frequency value of the maximum frequency gray scale level is larger when the gas-liquid mixing is uniform and the gray distribution curve of the image is more concentrated. When the two-phase mixing is nonuniform, the size of the liquid drops at different positions in the mixing zone is different, and the scattering effect is different, resulting in different gray levels of the pixels. When the twophase mixing is uniform, the scattering effect of the liquid drops at different positions in the mixing zone is the same, and the gray scale level of the pixels is almost the same. Therefore, when the two-phase mixing is uniform, the gray distribution curve of the two-phase flow image is more concentrated.

The above research shows that the difference of the flow pattern, the gas-liquid mixing intensity and mixing uniformity in the mixing zone can be described by the gray distribution of the image. Therefore, the influence of flow parameters and nozzle structure on the flow pattern, the gas-liquid mixing intensity and uniformity can be studied by gray distribution of the images. However, factors such as the position, number and illumination intensity of the experimental light source, as well as the preliminary processing of the experimental images will affect the gray distribution of the experimental images, so that the accuracy of using the gray distribution of the images to study the two-phase mixing is greatly reduced. Therefore, during the experiment in this paper, the relative arrangement of the light source and the image acquisition equipment is always maintained and the illumination intensity is constant. In addition, when using Matlab software to extract the gray distribution of the experimental images, we adopted the same parameters to ensure that the influence of image processing on the research results was minimized. On the whole, it is feasible to use gray distribution of the images to study two-phase mixing inside the nozzle. The control of the experimental method and the conditions can ensure the accuracy of the research results.

\section{Results and Discussions}

The purpose of this paper is to decode the gray information of the experimental images to study the two-phase flow in the mixing zone of the flow focusing/blurring nozzle. First, through the gray distribution of the experimental images, we qualitatively studied the influence of nozzle structure and flow parameters on the two-phase mixing inside the nozzle. Second, we use the gray distribution of the experimental images to analyze the strength of influence between different parameters on the two-phase mixing in the mixing zone. Last, we use a gray histogram to study and analyze the local two-phase flow field in the mixing zone.

\subsection{The Influence of Flow and Structure Parameters on Two-Phase Mixing Inside the Nozzle}

Experiments were carried out on the flow focusing/blurring nozzle under different conditions and the gray distribution of the two-phase flow images in the mixing zone of the nozzle was extracted and analyzed. Because we mainly study the gas-liquid mixing in the mixing zone, the conditions that make the flow blurring or close to the flow blurring inside 
the nozzle are selected in the experiment. Figure 7 shows the gray distribution curves of the two-phase flow images in the mixing zone under different water flow rates.

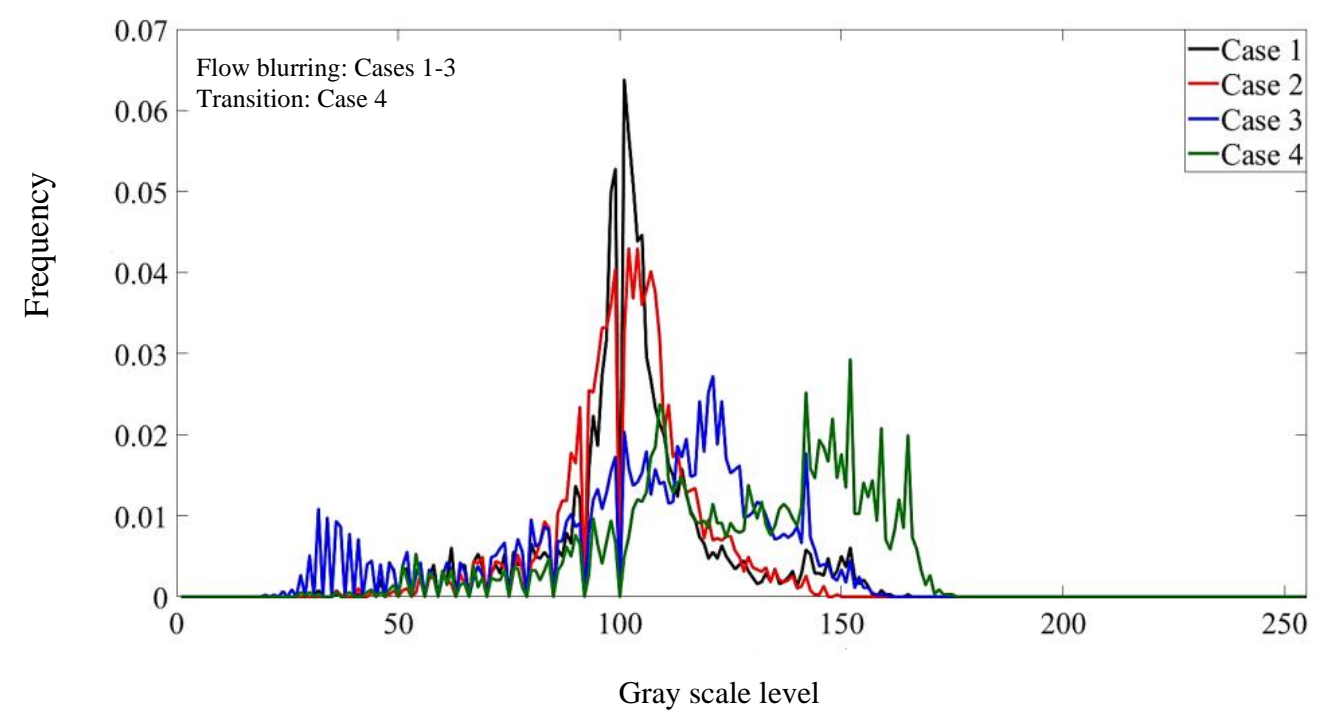

Figure 7. The gray distribution curves of the two-phase flow images under different water flow rates: the air flow rate, tube-hole distance and orifice diameter are $80 \mathrm{~L} / \mathrm{min}, 1 \mathrm{~mm}$ and $5 \mathrm{~mm}$, respectively. For cases 1 to 4 , the water flow rates are $100 \mathrm{~mL} / \mathrm{min}, 200 \mathrm{~mL} / \mathrm{min}, 400 \mathrm{~mL} / \mathrm{min}$ and $600 \mathrm{~mL} / \mathrm{min}$, respectively.

As can be seen from Figure 7, with the increase of the water flow rate, the gray scale level of the two-phase flow image in the mixing zone increases and the concentration of the gray distribution curve decreases. This indicates that the mixing intensity and uniformity of the gas and liquid in the mixing zone will be weakened with the increase of the water flow rate in a certain range. This indicates that the increase of the water inertia force is not conducive to the full mixing of the two-phase fluids in the nozzle.

Figure 8 shows the gray distribution curves of the two-phase flow images in the mixing zone under different air flow rates.

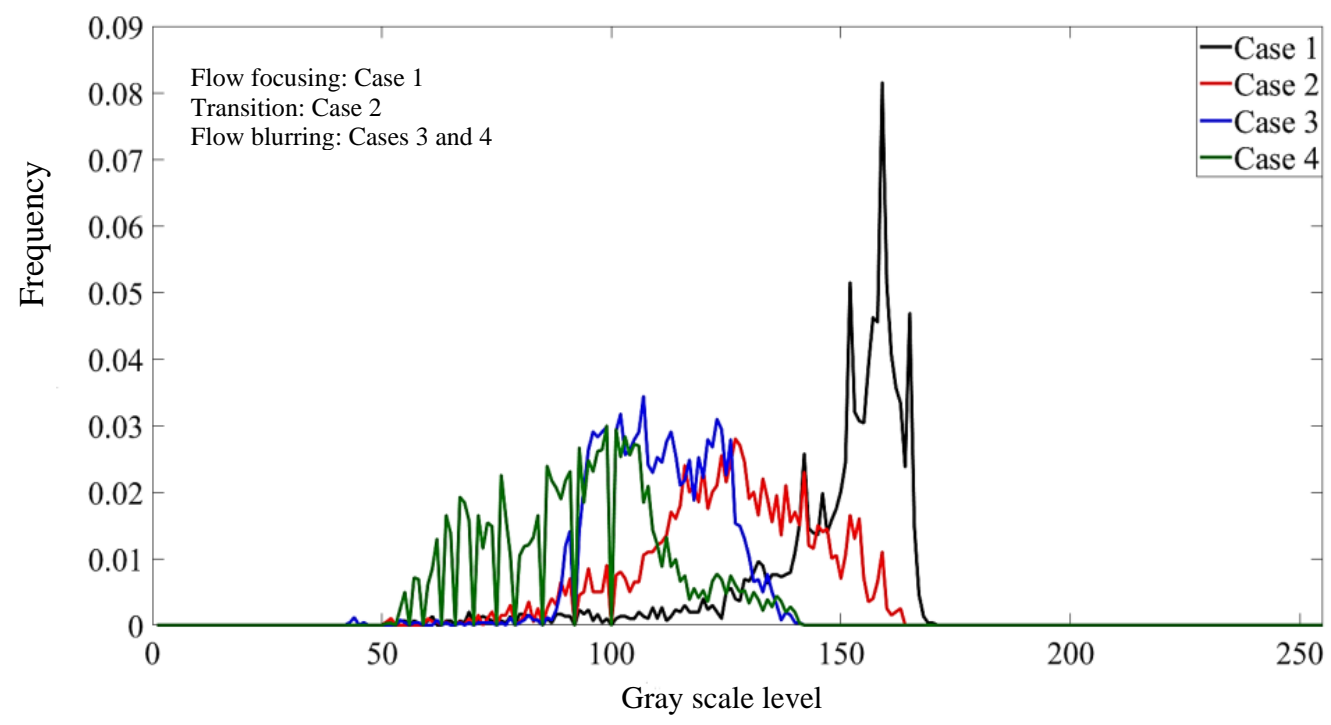

Figure 8. The gray distribution curves of the two-phase flow images under different air flow rates: the water flow rate, tube-hole distance and orifice diameter are $100 \mathrm{~mL} / \mathrm{min}, 1 \mathrm{~mm}$ and $5 \mathrm{~mm}$, respectively. For cases 1 to 4 , the air flow rates are $10 \mathrm{~L} / \mathrm{min}, 20 \mathrm{~L} / \mathrm{min}, 40 \mathrm{~L} / \mathrm{min}$ and $60 \mathrm{~L} / \mathrm{min}$, respectively. 
Figure 8 shows that with the increase of the air flow rate, the gray scale level of the two-phase flow image in the mixing zone decreases. When the air flow rate is small (such as in case 1), the flow pattern in the mixing zone is closer to flow focusing and the single-phase uniformity is better, so the concentration of the gray distribution curve is better. Therefore, it indicates that, with the increase of the air flow rate, the mixing intensity and uniformity of the two-phase will become stronger. Different from the water, the increase of the air inertia force can make the mixing effect of the gas-liquid phase better in the mixing zone.

Figure 9 shows the gray distribution curves of the two-phase flow images in the mixing zone under different tube-hole distances. As can be seen from the figure, the gray scale level of the two-phase flow image in the mixing zone decreases with the decrease of the tube-hole distance; when the flow pattern is flow blurring, the concentration of gray distribution curve will be enhanced with the tube-hole distance's decreases. In other words, the increase of the tube-hole distance will weaken the mixing intensity and uniformity of gas and liquid in the mixing zone. This indicates that the increase of the tube-hole distance is also not conducive to the full mixing of the two phases in the nozzle.

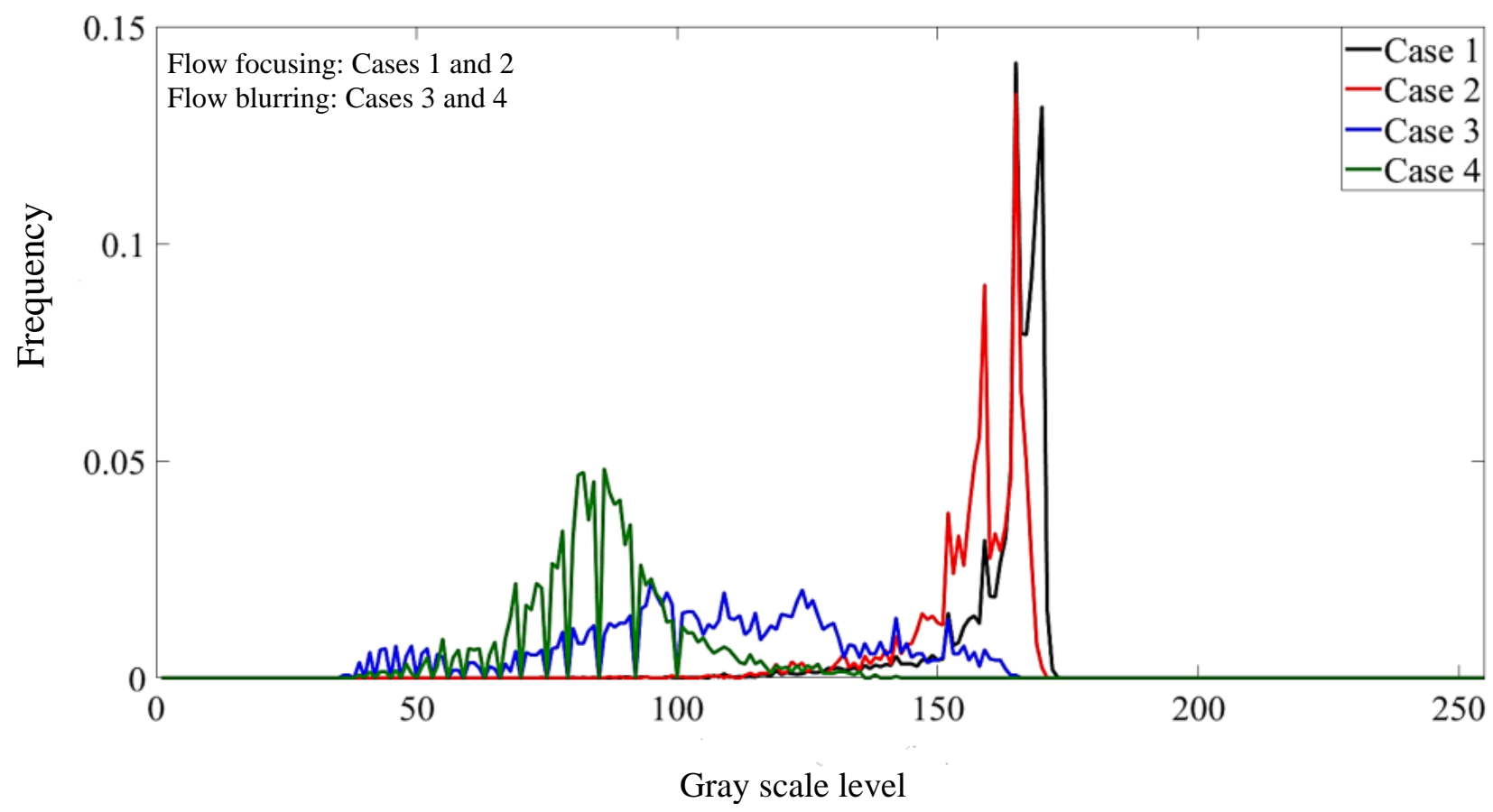

Figure 9. The gray distribution curves of the two-phase flow images under different tube-hole distances: the water flow rate, air flow rate and orifice diameter are $100 \mathrm{~mL} / \mathrm{min}, 100 \mathrm{~L} / \mathrm{min}$ and $5 \mathrm{~mm}$, respectively. For cases 1 to 4 , the tube-hole distance is $4 \mathrm{~mm}, 3 \mathrm{~mm}, 2 \mathrm{~mm}$ and $1 \mathrm{~mm}$, respectively.

Figure 10 shows the gray distribution curves of the two-phase flow images in the mixing zone under different orifice diameters. The influence of the orifice diameter on twophase mixing in a mixing zone is relatively simple. Figure 10 shows that, when the orifice diameter is larger than the inner tube, the two-phase mixing intensity and uniformity inside the mixing zone are weak. When the orifice diameter is equal to or less than the inner tube, the concentration of the gray distribution curve is significantly enhanced, which indicates that when the orifice diameter is equal to or less than the inner tube, the two-phase mixing in the mixing zone is significantly better than that when the orifice diameter is larger than the inner tube. When the orifice diameter is equal to or less than the inner tube, the mixing degree of the two phases in the mixing zone is not much different. On the whole, the increase of the orifice diameter is unfavorable to the good mixing of the two phases in the mixing zone. 


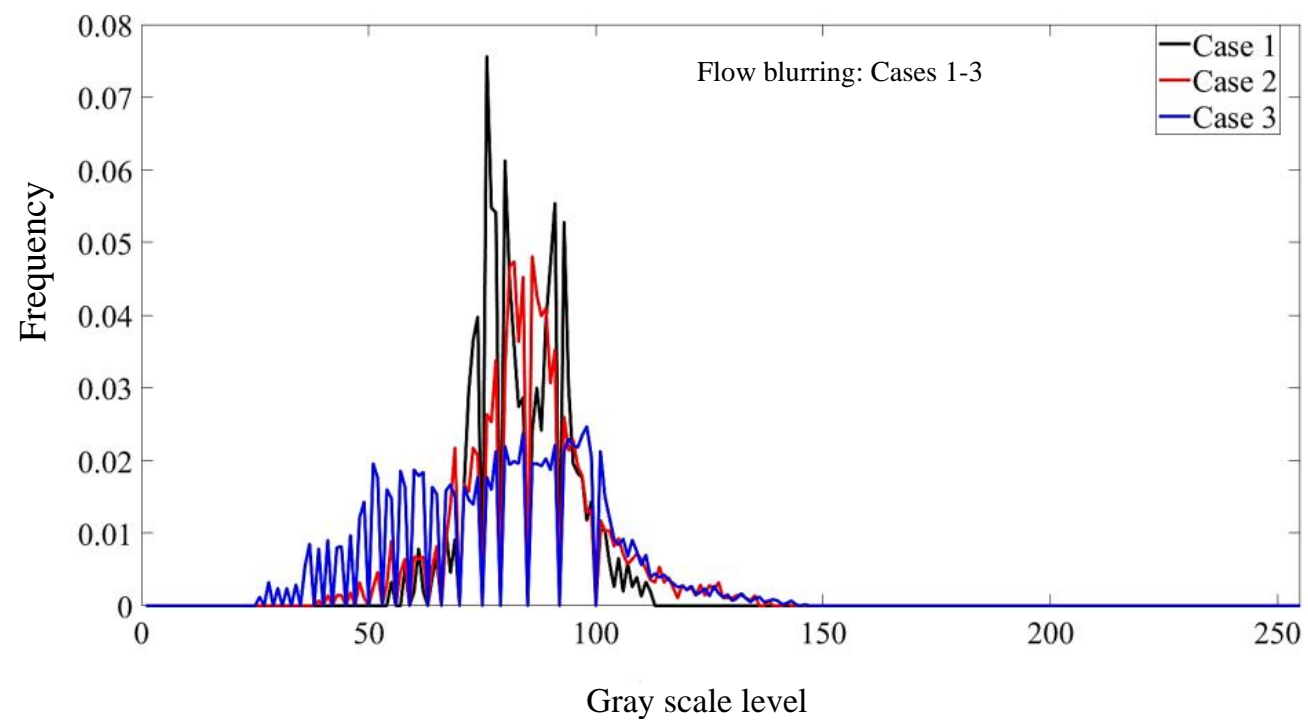

Figure 10. The gray distribution curves of the two-phase flow images under different orifice diameter: the water flow rate, air flow rate and tube-hole distance are $100 \mathrm{~mL} / \mathrm{min}, 100 \mathrm{~L} / \mathrm{min}$ and $1 \mathrm{~mm}$, respectively. For cases 1 to 3 , the orifice diameter is $3 \mathrm{~mm}, 5 \mathrm{~mm}$ and $7 \mathrm{~mm}$, respectively.

The above analysis shows that the influence of the water flow rate, the tube hole distance and the orifice diameter on two-phase mixing in a mixing zone is similar; with the increase of these parameters, it is not conducive to the full mixing of the two-phase in the nozzle. The influence of the air flow rate on the two-phase mixing in the mixing zone is completely opposite; the increase of the air flow rate will increase the intensity and uniformity of the two-phase mixing in the mixing zone.

\subsection{Comparison of the Influence of Different Parameters on Two-Phase Mixing}

The influence of the nozzle structure and flow parameters on two-phase mixing in a mixing zone is analyzed and discussed by using gray scale level distribution. In the nozzle design and practical application adjustment, in most cases, multiple parameters will change simultaneously to affect the operation of the flow focusing/blurring nozzle. Therefore, it is meaningful to study and compare the effects of different parameters on two-phase mixing in the mixing zone. In this section, the effects of different parameters on two-phase mixing in the mixing zone are compared and analyzed.

The effect of the liquid flow rate, the tube hole distance and the orifice diameter on gas-liquid mixing is similar, while the effect of the gas flow rate on gas-liquid mixing is opposite. Therefore, after increasing gas flow rate and liquid flow rate, gas flow rate and tube hole distance, gas flow rate and orifice diameter, in equal proportion, the influence degree of these four parameters on gas-liquid mixing can be qualitatively studied through the change of the gray distribution curve.

Figure 11 shows the gray distribution curves of the two-phase flow images in the mixing zone with different air and water flow rates. As can be seen from Figure 11, as the air flow rate and the water flow rate increases proportionally, the gray scale level of the two-phase flow image in the mixing zone increases slowly, indicating that the degree of two-phase mixing decreases. This indicates that the liquid inertia force has a slightly stronger effect on the two-phase mixing in a mixing zone than the gas inertia force. This is due to the difference in inertia forces between the gas and liquid: when the gas-liquid phase is mixing in the mixing zone, although the gas velocity is higher than the liquid, the water density is much higher than air, so as the two-phase flow rate increases proportionally, the air inertia force will be lower than water. Therefore, the effect of the liquid flow rate on two-phase mixing is slightly greater than gas flow rate. 


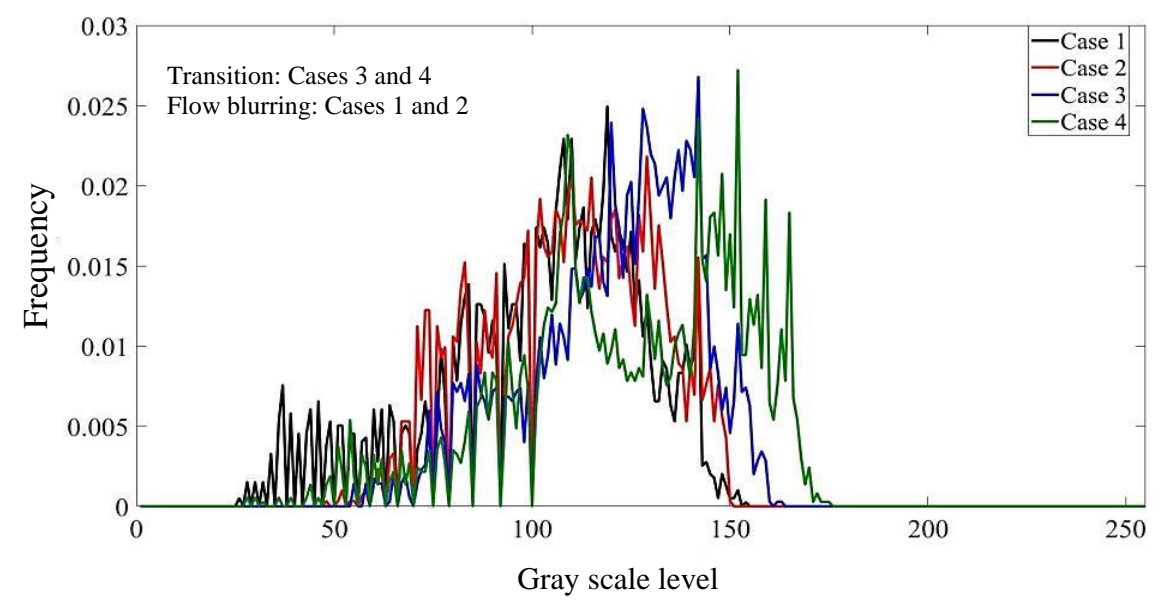

Figure 11. The gray distribution curves of the two-phase flow images under different air and water flow rates: the tube-hole distance and orifice diameter are $1 \mathrm{~mm}$ and $5 \mathrm{~mm}$. Based on case 1, the air and water flow rates in cases 1 to 4 can be dimensionless into (in the form of air flow rate/water flow rate): $1 / 1,1.5 / 1.5,2 / 2$ and $2.5 / 2.5$.

Figure 12 shows the gray distribution curves of the two-phase flow images in the mixing zone under different air flow rates and tube hole distances. As can be seen from the figure, when the air flow rate and the tube hole distance increase proportionally, the gray scale level of the two-phase mixing image does not change much at the beginning, and then the gray scale level of the image increases significantly. The air velocity in the mixing zone will decrease with the increase of the tube hole distance, thus reducing the mixing degree of gas and liquid in the nozzle. At the initial stage, when the air flow rate and the tube hole distance increase proportionally, the decrease in air velocity caused by the increase of the tube hole distance in the mixing zone is compensated by the increase of the air flow rate, so the gray scale level of the two-phase mixing image does not change much. However, when the tube hole distance is large, the increase of the air flow rate is not enough to make up for the air velocity decrease in the mixing zone, resulting in a significant decrease in the degree of the two-phase mixing. Therefore, when the tube hole distance is small, the air flow rate and the tube hole distance have the same effect on the two-phase mixing, while when the tube hole distance is large, the effect of the tube hole distance on two-phase mixing is more obvious than the gas flow rate.

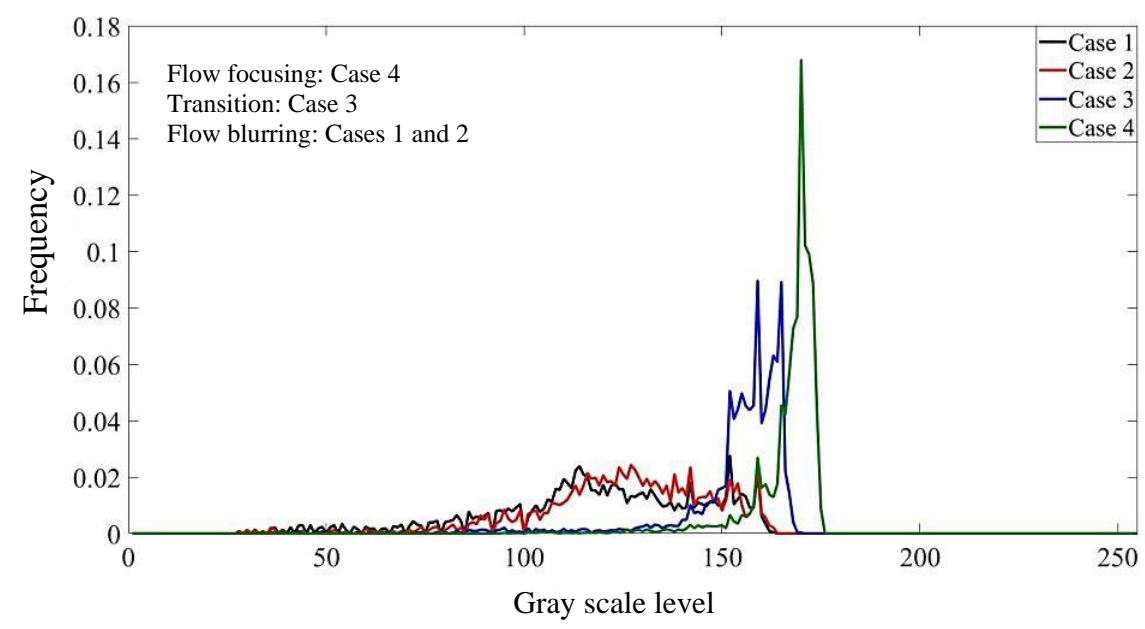

Figure 12. The gray distribution curves of the two-phase flow images under different air flow rate and tube hole distances: the water flow rate and orifice diameter are $100 \mathrm{~mL} / \mathrm{min}$ and $5 \mathrm{~mm}$. Based on case 1 , the air flow rate and tube hole distances in cases 1 to 4 can be dimensionless into (In the form of air flow rate/tube hole distance):1/1,2/2,3/3 and 4/4. 
Figure 13 shows the gray distribution curves of the two-phase flow images in the mixing zone under different air flow rates and orifice diameters. Due to the limitation of nozzle processing conditions, we failed to increase the gas flow rate and the orifice diameters proportionally in the experiment. When the orifice diameter grows faster than the air flow rate, the gray scale level of the two-phase flow image still decreases, indicating that the effect of the air flow rate on two-phase mixing is stronger than the orifice diameter. The orifice diameter also affects two-phase mixing by affecting the gas velocity in the mixing zone but, because the orifice is downstream of the mixing zone, the effect of the orifice diameter on the two-phase flow in the mixing zone is limited.

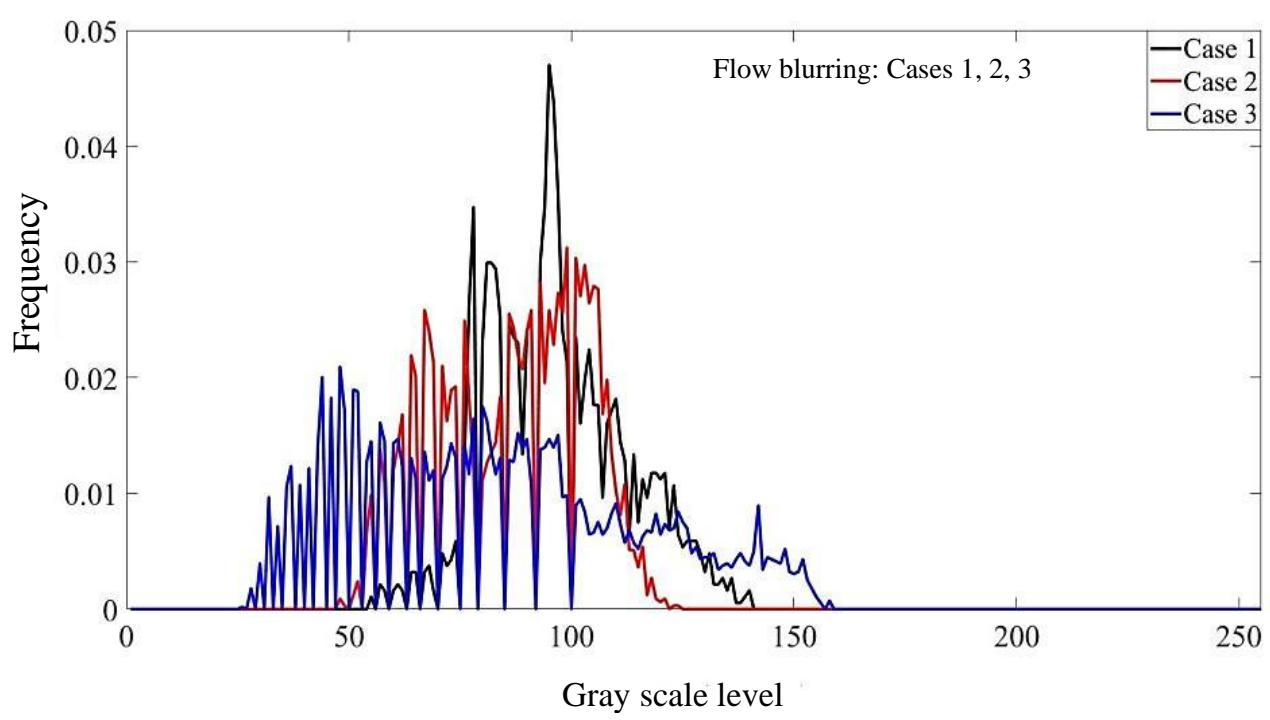

Figure 13. The gray distribution curves of the two-phase flow images under different air flow rate and orifice diameter: the water flow rate and tube hole distance are $100 \mathrm{~mL} / \mathrm{min}$ and $1 \mathrm{~mm}$. Based on case 1 , the air flow rate and orifice diameter in cases 1 to 3 can be dimensionless into (In the form of air flow rate/orifice diameter):1/1, 1.5/1.66 and 2/2.33.

The research and analysis shows that, compared with other factors (the water inertia force or the tube-hole distance), the effect of the air inertia force on two-phase mixing is weak. This also explains why flow blurring usually occurs under high gas flow rate. When the flow pattern in the mixing zone is close to flow blurring or just reaches flow blurring, the atomization effect of the nozzle on the liquid is more ideal. Therefore, under the condition that the requirements are met, the air flow rate can be reduced, thus reducing the energy consumption during the use of the flow blurring nozzle.

\subsection{Local Analysis of Two-Phase Flow Field in Mixing Zone}

Although we have studied two-phase flow and mixing in the mixing zone under different nozzle structures and flow parameters by using the gray distribution curve, as the gray distribution curve is a frequency statistical result, we do not know much about the two-phase local flow field in the mixing zone. The local flow field information is important to understand the two-phase interaction in the mixing zone. Therefore, we used Matlab software to analyze the local flow field in the mixing zone with different flow patterns.

Figure 14 shows the radial and axial gray scale distribution of the local flow field under different flow patterns in the mixing zone. Figure 14a shows the gray scale distribution of the radial flow field in the center of the mixing zone under different flow patterns. Figure $14 \mathrm{~b}$ shows the gray scale distribution of the axial flow field in the center of the mixing zone under different flow patterns. 

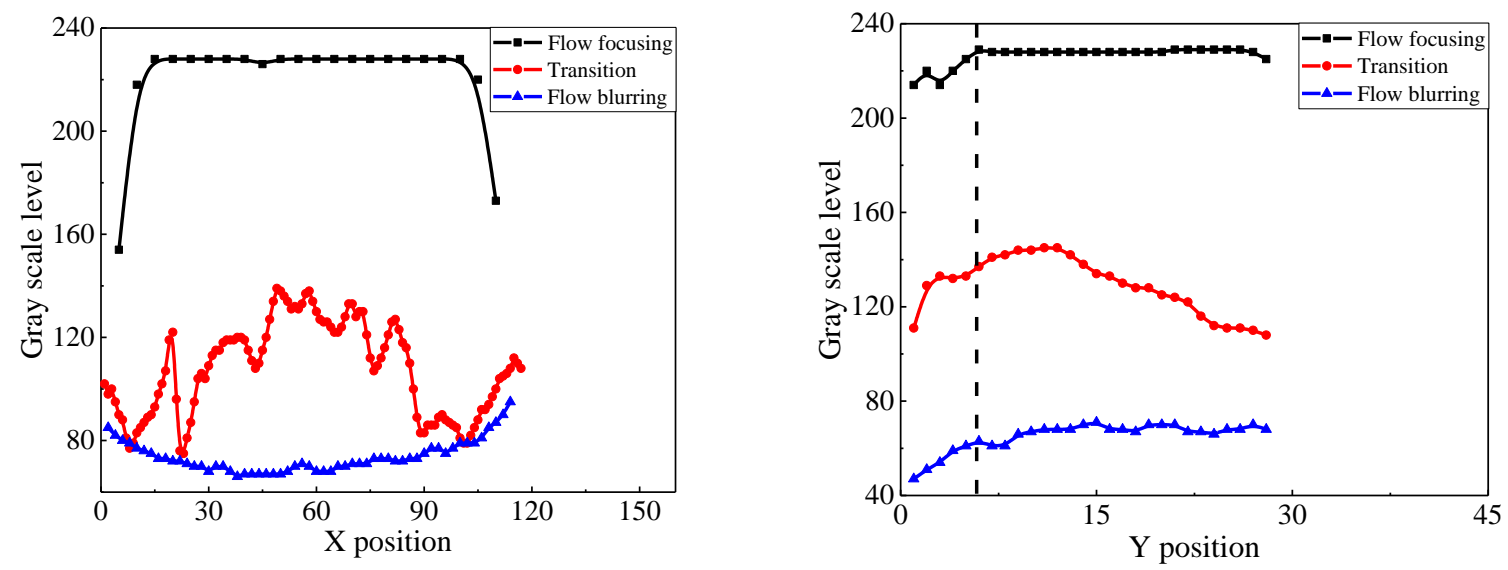

Figure 14. Gray scale distribution of local flow field.

As can be seen from Figure 14a, the gray scale level of the radial flow field is larger, and the gray distribution is more uniform during flow focusing. The obvious gas-liquid interface makes the gray scale level of the flow field lower when the radial position is smaller or larger. When the flow pattern is flow blurring, the gray scale level of the radial flow field in the mixing zone is low, but the gray distribution is still relatively uniform. The thickness of the two-phase mixture center region is relatively large, which results in the lower gray scale level of the flow image in the radial center region but actually the radial gray scale distribution is relatively uniform. In the transition mode, the gray scale level of the radial flow field is close to that of the flow blurring, but the gray distribution curve is very nonuniform. This is due to the instability caused by the strong radial inertia force of the gas acting on the liquid.

In general, the gas-liquid flow or mixing in the mixing zone is stable in the radial direction when the flow pattern is flow focusing or flow blurring, while the interaction of the gas-liquid two-phase radial inertia force in the transition mode makes the gas-liquid two-phase mixing radially unstable.

As shown in Figure 14b, for different flow patterns, when the axial distance is small, the gray scale level of the two-phase flow images will increase with the increase of the axial position. This is because the liquid column or liquid mist in this area will reflect the inner tube wall, thus affecting the gray scale level of the flow images. In the flow focusing mode, the gray scale level of the axial flow field is high and uniform. In the flow blurring mode, the gray scale level of the axial flow field is lower, and the gray scale level distribution curve has small pulsation. In the transition mode, the gray scale level of the axial flow field is close to the flow blurring mode and the gray scale level distribution curve decreases in pulsation. The analysis shows that the gas-liquid flow in the mixing zone is axially stable when in flow focusing mode, while the gas-liquid two-phase flow in the mixing zone is axially unstable when transition mode or flow blurring mode, and the gas-liquid flow characteristics are similar in these two modes.

The analysis of the local flow field in the mixing zone shows that the gas-liquid flow in the mixing zone is completely stable in the flow focusing mode. In the flow blurring mode, the gas-liquid flow in the mixing zone is stable in the radial direction, but there are small pulsations in the axial direction. In the transition mode, the gas-liquid flow in the mixing zone is unstable, and its flow characteristics are close to the flow blurring mode.

\section{Conclusions}

(1) The feasibility of using the gray distribution curve of the gas-liquid flow images to study the two-phase flow in the mixing zone of the flow focusing/blurring nozzle is analyzed. Preliminary analysis shows that the gray distribution of the flow images can represent the mixing intensity and uniformity of the gas and liquid in the mixing zone; 
(2) The influence of different parameters on gas-liquid mixing was studied by using the gray distribution of the experimental images. The results show that the increase of the liquid flow rate, the tube hole distance and the orifice diameter is not conducive to the two-phase mixing in the mixing zone, while the increase of the gas flow rate is conducive to the full mixing of the gas and liquid in the mixing zone;

(3) The effects of different parameters on two-phase mixing in the mixing zone were compared by using the gray distribution curve of the experimental images. The results show that the influence of the gas inertia force on two-phase mixing is weaker than the liquid inertia force. At low flow rate, the influence of the gas inertia force is similar to the tube hole distance. The orifice diameter has a certain effect on the two-phase mixing, but the effect is weak;

(4) The gas-liquid flow field in the mixing zone of the flow focusing/blurring nozzle under different modes is analyzed and studied by using the gray distribution curves. The results show that the two-phase flow in the mixing zone is stable in the flow focusing mode. In the flow blurring mode, the two-phase mixing in the mixing zone is stable in the radial direction and pulsating in the axial direction. In the transition mode, the two-phase flow is unstable, but its flow characteristics are close to the flow blurring mode.

Author Contributions: Conceptualization, J.Z. and M.L.; methodology, Z.N.; software, C.S.; experiment, J.Z. and C.S.; data curation, J.Z.; writing — original draft preparation, J.Z.; writing—review and editing, M.L. and Z.N. All authors have read and agreed to the published version of the manuscript.

Funding: This research was funded by the National Natural Science Foundation of China, grant numbers 51776016 and 52006136, Beijing Natural Science Foundation, grant number 3182030, and the National Key Research and Development Program, grant number 2017 YFB0103401.

Institutional Review Board Statement: Not applicable.

Informed Consent Statement: Not applicable.

Data Availability Statement: The data that support the findings of this study are available upon reasonable request.

Conflicts of Interest: The authors declare that they have no conflict of interest.

\section{References}

1. Ganan-Calvo, A.M. Generation of steady liquid microthreads and micron-sized monodisperse sprays in gas streams. Phys. Rev. Lett. 1998, 80, 285-288. [CrossRef]

2. Ganan-Calvo, A.M. Enhanced liquid atomization: From flow-focusing to flow-blurring. Appl. Phys. Lett. 2005, 86, 4601. [CrossRef]

3. Si, T.; Yin, X.Z. Progress and application of flow focusing. Chin. Sci. Bull. 2011, 56, 537-546. [CrossRef]

4. Ganan-Calvo, A.M.; González-Prieto, R.; Riesco-Chueca, P.; Herrada, M.A.; Flores-Mosquera, M. Focusing Capillary Jets Close to the Continuum Limit. Nat. Phys. 2007, 3, 737-742. [CrossRef]

5. Vega, E.J.; Montanero, J.M.; Herrada, M.A.; Ganan-Calvo, A. Global and local instability of flow focusing: The influence of the geometry. Phys. Fluids 2010, 22, 064105. [CrossRef]

6. Montanero, J.M.; Muñoz, N.R.; Herrada, M.A.; Ganan-Calvo, A. Global stability of the focusing effect of fluid jet flows. Phys. Rev. E 2011, 83, 036309. [CrossRef] [PubMed]

7. Herrada, M.A.; Ganan-Calvo, A.; Ojeda-Monge, A.; Bluth, B.; Riesco-Chueca, P. Liquid flow focused by a gas: Jetting, dripping, and recirculation. Phys. Rev. E 2008, 78, 036323. [CrossRef] [PubMed]

8. Si, T.; Li, F.; Yin, X.-Y. Modes in flow focusing and instability of coaxial liquid-gas jets. J. Fluid Mech. 2009, 629, 1-23. [CrossRef]

9. Jiang, L.; Agrawal, A.K. Investigation of Glycerol Atomization in the Near-Field of a Flow-Blurring Injector using Time-Resolved PIV and High-Speed Visualization. Flow Turbul. Combust. 2015, 94, 323-338. [CrossRef]

10. Jiang, L.L. Investigation of Atomization Mechanisms and Flame Structure of a Twin-Fluid Injector for Different Liquid Fuels. Ph.D. Thesis, The University of Alabama, Tuscaloosa, AL, USA, 2014.

11. Simmons, B.M.; Agrawal, A.K. Flow Blurring Atomization for Low-Emission Combustion of Liquid Biofuels. Combust. Sci. Technol. 2012, 184, 660-675. [CrossRef]

12. De Azevedo, C.G.; Andrade, J.C.D.; Costa, F.D.S. Characterization of a blurry injector for burning biofuels in a compact flameless combustion chamber. In Proceedings of the 14th Brazilian Congress of Thermal Sciences and Engineering, Rio de Janeiro, Brazil, 18-22 November 2012.

13. Xiao, Q.-T.; Pan, J.-X.; Xu, J.-X.; Wang, H.; Lv, Z.-H. Hypothesis-testing combined with image analysis to quantify evolution of bubble swarms in a direct-contact boiling heat transfer process. Appl. Therm. Eng. 2017, 113, 851-857. [CrossRef] 
14. Xiao, Q.; Yang, K.; Wu, M.; Pan, J.; Xu, J.; Wang, H. Complexity evolution quantification of bubble pattern in a gas-liquid mixing system for direct-contact heat transfer. Appl. Therm. Eng. 2018, 138, 832-839. [CrossRef]

15. Xiao, Q.; Luo, W.; Huang, J.; Xu, J.; Wang, H. Interplay of fluids mixing and heat transfer in a dual-loop ORC direct contact heat exchanger used for waste heat utilization. Inst. Mech. Eng. Part C J. Mech. Eng. Sci. 2020, 234, 2294-2305. [CrossRef]

16. Wang, C.; Yang, P.Y.; Xu, J.X.; Wang, S.B.; Wang, H. Study on flow regime identification of gas-liquid two phase flows in the mixing process by top blowing. J. Kunming Univ. Sci. Technol. 2014, 39, 77-82.

17. Yang, L. Study of Gray Feature of Water Bubbles Group Image. Master's Thesis, Harbin Engineering University, Harbin, China, 2015.

18. Li, X.; Liu, N.; Hao, P.; Zhang, X.; He, F. Screech feedback loop and mode staging process of axisymmetric underexpanded jets. Exp. Therm. Fluid Sci. 2021, 122, 110323. [CrossRef]

19. Li, X.-R.; Zhang, X.-W.; Hao, P.-F.; He, F. Acoustic feedback loops for screech tones of underexpanded free round jets at different modes. J. Fluid Mech. 2020, 902. [CrossRef]

20. Semlitsch, B.; Malla, B.; Gutmark, E.J.; Mihăescu, M. The generation mechanism of higher screech tone harmonics in supersonic jets. J. Fluid Mech. 2020, 893. [CrossRef]

21. Gonzalez, R.C.; Woods, R.E. Digital Image Processing, 4th ed.; Pearson: London, UK, 2017. 